\title{
SNR POLARIZATION AND THE DIRECTION \\ OF THE MAGNETIC FIELD
}

\author{
D.K. Milne \\ Division of Radiophysics, CSIRO \\ PO Box 76 Epping, NSW 2121, Australia
}

\begin{abstract}
At the CSIRO Division of Radiophysics we are currently engaged in a program to map polarization in SNRs at $8.4 \mathrm{GHz}$. These results are compared with earlier Parkes $5 \mathrm{GHz}$ maps to deduce the direction of magnetic field, Faraday rotation and depolarization.
\end{abstract}

Over the past decade or so linear polarization observations have been published for $\sim 70$ supernova remnants. We accept that this polarized radio emission is generated by the synchrotron process, which implies a magnetic field directed normal to the intrinsic electric vector. For a uniform magnetic field the degree of polarization is independent of frequency and is given in terms of the spectral index $(\alpha)$ by

$$
P=(3-3 \alpha) /(5-3 \alpha), \quad \text { i.e. } P=0.7 \text { for } \alpha=-0.5 \text {. }
$$

If the magnetic field is not uniform throughout the emission region the degree of polarization is reduced, and if the magnetic field is completely random it is zero. The observed polarization will be decreased even in the presence of uniform magnetic fields by differential Faraday rotation of emission from regions at different depths. The observed polarization may be further reduced by Faraday rotation across the telescope beam.

Faraday rotation of the polarization position angle is generally observed and, in summary, the position angle should vary proportionally to wavelength squared if the rotation is outside the emitting region and will follow a more complicated relationship if it is inside.

Measurements of the distribution of polarization, Faraday rotation, depolarization and the direction of the magnetic field could tell us much about the energy process and evolution of these objects.

At the CSIRO Division of Radiophysics we are mapping SNR polarization at $8.4 \mathrm{GHz}$ ( $3^{\prime}$ arc beam) and comparing these with the Parkes 5 $\mathrm{GHz}$ maps (Milne and Dickel, 1975) to obtain the distribution of rotation measure, depolarization and direction of the projected magnetic field over the remnants; the first results, for the remnants G291.0-0.1 and G7.7-3.7, have been published (Roger et al., 1986 and Milne et al., 1986 respectively). A further 10 fields are currently being investigated. 
In Figure 1 we illustrate this work with a preliminary map of G316.30.0 , which shows the projected directions of the magnetic field, deduced from 5 and $8.4 \mathrm{GHz}$ measurements, superimposed on an $843 \mathrm{MHz}$ Molonglo synthesis map (Milne et al., 1987).

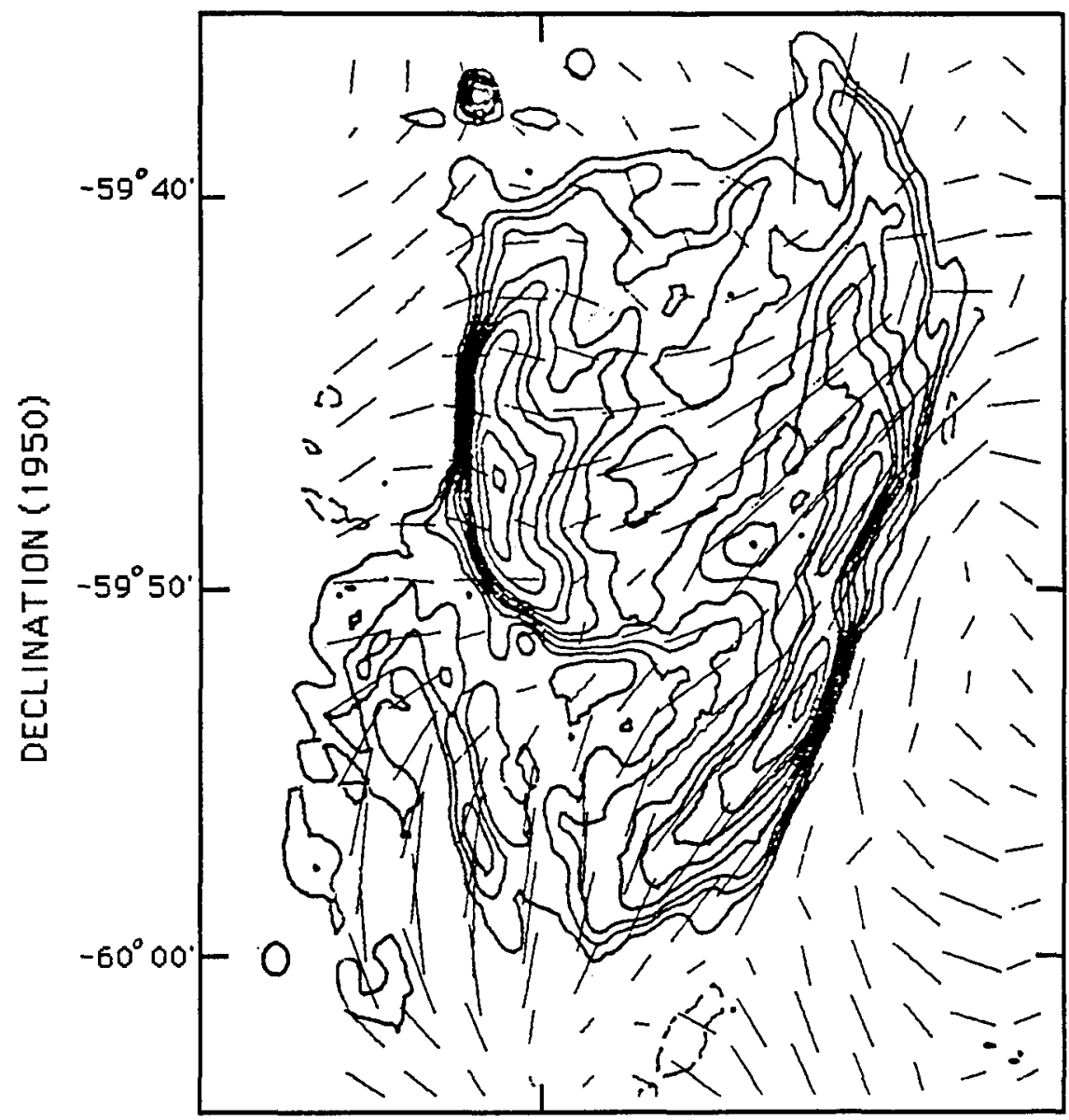

$14 \mathrm{~h} 38 \mathrm{~m}$

RIGHT ASCENSION (1950)

Fig. 1 - Direction of the projected magnetic field in the SNR G316.3-0.0 deduced from 5 and $8.4 \mathrm{GHz}$ polarization. The magnitude of these vectors indicates the mean polarization intensities at these two frequencies. The vectors are shown superimposed on the $843 \mathrm{MHz}$ Molonglo total power map (Milne et al., 1987). 
At present there are 27 SNRs for which polarization observations have been published - sufficient to deduce the direction of the projected magnetic field over the remnant. These results have been collated to form "An Atlas of SNR Magnetic Fields" (Milne, 1987). In Table 1 we list these remnants, together with their ages, structural form and the suggested direction of the projected magnetic field.

Table 1 SNRs with observed magnetic field directions

\begin{tabular}{|c|c|c|c|}
\hline Remnant & Age (yrs)* & Structural form & Magnetic field direction \\
\hline G5.4-1.0 & 2500 & Shell & Radial in northern arc \\
\hline G6.4-0.1 & 6000 & Shell & Tangential \\
\hline G7.7-3.7 & 2000 & Shell & Across shell \\
\hline G18.8+0.3 & 4000 & Shell & Radial \\
\hline G21.5-0.9 & 500 & Filled + shell(?) & Radial \\
\hline G21.8-0.6 & 4000 & Shell & $?$ \\
\hline G34.6-0.5 & 2500 & Shell & Radial (?) \\
\hline G74.0-8.6 & 12000 & Shell + blow-out (?) & Tangential in blow-out \\
\hline G89.0+4.7 & 7000 & Shell? & \\
\hline$G 93.3+6.9$ & 1500 & Shell & Tangential \\
\hline G111.7-2.1 & 300 & Shell & Radial \\
\hline G120.1+1.4 & $413^{*}$ & Shell & Radial \\
\hline G127.1+0.5 & 18000 & Shell & Tangential \\
\hline $\mathrm{G} 130.7+3.1$ & $805^{*}$ & Filled & Longitudinal \\
\hline G184.6-5.8 & $932^{*}$ & Filled & Radial on periphery \\
\hline G189.1+2.9 & 2500 & Shell & Radial \\
\hline G260.4-3.4 & $3000 ?$ & Shell & Radial \\
\hline G263.9-3.0 & 11000 & Shell & $?$ \\
\hline G291.0-0.1 & 4000 & Filled + faint shell & Directed along central bar \\
\hline G296.5+10.0 & 5000 & Shell & Tangential \\
\hline G315.4-2.3 & $1800^{*}$ & Shell & Radial \\
\hline G316.3-0.0 & 700 & Shell + blow-out (?) & Radial in shell \\
\hline G320.4-1.2 & 1600 & Shell & Radial \\
\hline G326.3-1.8 & 3000 & Faint shell + bright region & $?$ \\
\hline $\mathrm{G} 327.6+14.0$ & $980^{*}$ & Shell & Radial \\
\hline $\mathrm{G} 327.4+0.4$ & 6000 & Shell(s) & $?$ \\
\hline $\mathrm{G} 332.4+0.1$ & 5000 & Shell + blow-out (?) + jet & Tangential \\
\hline
\end{tabular}


The conclusions that can be drawn from our current knowledge can be summarized:

(a) A general large-scale field is readily seen at low resolutions, but at higher resolutions this may be concealed by increased detail.

(b) Radial fields are more prevalent than tangential fields.

(c) Radial fields predominate in the young remnants. All objects for which we have a definite (and consequently young) age have a field that is radial around the periphery; this is as we would expect it to be, since firstly, the young SNR has not been broken up as much by interaction with the ISM, and secondly, a radial field in a spherical shell is seen as a radial field in all projections.

(d) Blowouts have possibly occurred in G74.0-8.6 (the Cygnus Loop), G316.3-0.0 (MSH 14-57) and in G3324+0.1 (Kes 32). In these objects and perhaps others it appears that an otherwise spherical shell has been breached, dragging the magnetic field into the blowout at this point (e.g. the most southern part of G316.3-0.0 in Figure 1).

(e) In spite of the preference for alignment of the brightest parts of a remnant nearest the galactic plane (Caswell, 1977) there seems to be no evidence for any preferred orientation in the direction of magnetic field.

Finally, there does not appear to be an answer yet to the question whether polarization or the magnetic field direction exhibits any interaction with the ISM or with the interstellar magnetic field.

\section{References}

Caswell, J.L. (1977). Proc. Astron. Soc. Aust. 3, 130.

Caswell, J.L., and Lerche, I. (1979). Mon. Not. R. Astron. Soc. 187, 201.

Milne, D.K. (1979). Aust. J. Phys. 32, 83.

Milne, D.K. (1987). "An atlas of SNR magnetic fields", Aust. J. Phys. (in press).

Milne, D.K., and Dickel, J.R. (1975). Aust. J. Phys. 28, 209.

Milne, D.K., Roger, R.S., Kesteven, M.J., Haynes R.F., Wellington, K.J., and Stewart, R.T. (1986). Mon. Not. R. Astron. Soc. 223, 487.

Milne, D.K., Roger, R.S., Kesteven, M.J., Haynes R.F., and Wellington, K.J. (1987) (in preparation).

Roger, R.S., Milne, D.K., Caswell, J.L., and Little, A.G. (1986). Mon. Not. R. Astron. Soc. 219, 8. 